Bangladesh J. Bot. 49(2): 197-204, 2020 (June)

\title{
IN VITRO SCREENING OF FUNGICIDES AND PLANT EXTRACTS AGAINST SIX PATHOGENIC FUNGI ISOLATED FROM COTTON (GOSSYPIUM ARBOREUM L.) SEED
}

\author{
Mst Naznin Nahar ${ }^{1}$ and Shamim Shamsi* \\ Department of Botany, University of Dhaka, Dhaka-1000, Bangladesh
}

Keywords: In vitro screening, Fungicides, Plant extracts, Cotton seed, Gossypium arboreum

\begin{abstract}
Six pathogenic fungi, namely Aspergillus flavus Link., A. niger van Tieghem (Type-I)., Curvularia lunata (Wakker) Boedijn, Fusarium moniliforme var. subglutinans Wr. \& Reink., Fusarium sporotrichioides Sherb., Mem. and Rhizoctonia solani J.G. Kuhn were isolated from cotton seeds. Five fungicides viz., Acrobat MZ, Autostin 50 WDG, Capvit 50 WP, Nativo 75 WP and Thiovit 80 WG were selected to evaluate in vitro efficacy at 100,200, 300, 400 and $500 \mathrm{ppm}$ concentrations against pathogenic fungi following poisoned food technique. Out of 5 fungicides Nativo 75 WP showed the complete growth inhibition of above mentioned six pathogenic fungi at all the used concentrations. Autostin 50 WDG showed complete growth inhibition of all tested pathogenic fungi except Curvularia lunata. Leaf extract of five angiospermic plants viz., Adhatoda vasica, Aegle mermelos, Azadirachta indica, Datura metel and Psidium guajava were selected to evaluate in vitro fungitoxicity at 5, 10, 15 and $20 \%$ concentrations against the test pathogens. At $20 \%$ concentration, out of the 5 plants extracts $A$. indica was found to be most active to inhibit the growth of Aspergillus niger (Type-I) (65.56\%) and Fusarium moniliforme var. subglutinans (75.00\%), Psidium guajava were most active against A. flavus $(81.29 \%)$ and Curvularia lunata $(72.23 \%)$, and Datura metel was most active against Fusarium sporotrichioides (64.77\%) and Rhizoctonia solani (42.44\%).
\end{abstract}

\section{Introduction}

Cotton (Gossypium spp.) is the most important natural textile fiber and vegetable oil source of the world (Vollmann and Laimer 2013). The genus Gossypium comprises around 50 species (Wendel et al. 2009) of which G. hirsutum, G. barbadense, G. arboreum and G. herbaceum are cultivated commercially throughout the world. Gossypium arboreum is grown in hilly regions of Bangladesh mainly in Chittagong and the Chittagong hill tracts. Economically it is very important as the lint is of superior quality, its staple is coarse and very short but very strong (Pandey 1980). More than 80 countries in the world and $2.5 \%$ cultivable lands are being used to cultivate cotton Nearly $80 \%$ of garments made in Bangladesh are sourced from cotton (Uddin and Mortuza 2015). Due to the rapid flourishing domestic weaving and knitting industries in Bangladesh, the overall consumption of cotton is also increasing enormously day by day. According to Bangladesh Textile Mills Association (BTMA), Bangladesh now imports approximately $\$ 2$ billion worth of cotton every year.

Every year the yield of cotton production is decreased by different seedling disease which are mainly caused by fungi, bacteria and viruses. Seedling diseases are generally caused by Thielaviopsis spp., Rhizoctonia spp., Pythium spp. and Fusarium spp. (Johnson and Chambers 1973). To mitigate the risk associated with fungal infection in cotton seeds, fungicides have been recommended (Hillocks et al. 1988, McLean and Gazaway 2000). Deposition of the residual fungicides in the soil, in plants and their fruits can negatively affect both the environment and human population in the long run. To minimize the environmental contamination and produce biologically safer fungicides, many alternatives have been tried, including physical treatments,

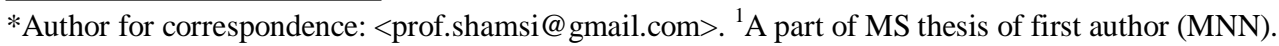


biological control, use of low toxicity chemicals such as plant extracts (Shahnaz et al. 2010, Sayago et al. 2012). Among them, the use of plant extracts has shown promising effects due to minimal environmental impacts and little to no danger to the consumers (Varma et al. 1999). Due to cost effectiveness and easy to make, plant extracts are gaining a lot of attention these days. Over the last three decades some articles related to the control of Fusarium species on various plants extract has been published (So 1990, Bansal and Rajesh 2000).

Present investigation was undertaken to screening of some selected fungicides and fungitoxicity of selected plant extracts against the pathogenic fungi isolated from cotton seeds.

\section{Materials and Methods}

Three varieties of cotton seeds, namely HC-1, HC-2 and HC-3 were collected from Cotton Development Board (CDB), Khamarbari, Farmgate, Dhaka. The collected seed samples were kept in polythene bag with airtight container in two conditions, one in room temperature $\left(25^{\circ} \mathrm{C}\right)$ and other in refrigerator at $4^{\circ} \mathrm{C}$ temperature for subsequent use. Fungi associated with cotton seeds were isolated following 'Blotter' and 'Tissue Planting' methods (Shamsi et al. 2010). The experiment was conducted in the laboratory of Mycology and Plant Pathology, Department of Botany, University of Dhaka, Bangladesh.

Identification of the isolated pathogenic fungi was determined following standard literatures (Thom and Rapper 1945, Benoit and Mathur 1970, Booth 1971, Barnett and Hunter 2000). Pathogenicity test of the isolated fungi were done following 'seed inoculation technique' (Chowdhury et al. 2015).

Five fungicides with different active ingredients viz., Acrobat MZ (60\% mancozeb and 9\% dimethamorph), Autostin 50 WDG (50\% carbendazim), Capvit 50 WP (copper oxychloride), Nativo 75 WP (tebuconazole and trifloxystrobin) and Thiovit 80 WG (sulphur) were collected from the Siddique Bazar, Gulistan, Dhaka. In vitro fungitoxicity of these fungicides at 100, 200, 300, 400 and 500 ppm concentrations was evaluated against Aspergillus flavus, Aspergillus niger (Type-I), Curvularia lunata, Fusarium moniliforme var. subglutinans, Fusarium sporotrichioides and Rhizoctonia solani.

For each fungicide, a stock solution having the concentration of 10,000 ppm was prepared. The calculated amount of stock solution of a fungicide was supplemented with sterilized PDA medium to get the concentration of 100, 200, 300, 400 and $500 \mathrm{ppm}$, respectively. The concentrations of fungicides were expressed in terms of its active ingredients. In control set, required amount of sterilized water instead of fungicide solution was added to the PDA medium. Then $15 \mathrm{ml}$ of medium was poured in each Petri plate and allowed them to solidify.

Therefore, at the center of the plate $5 \mathrm{~mm}$ agar disk of test pathogen was inoculated. Three replications were maintained in each treatment. The plates were incubated at $25 \pm 2^{\circ} \mathrm{C}$ in an incubator. The radial growth of control and treatment plates were measured at 5 days of incubation.

Fresh leaves of five angiospermic plants, namely Adhatoda vasica Nees., Azadirachta indica A. Juss., Datura metel L., Aegle mermelos L. and Psidium gujava L. were selected for evaluating their efficacy on the radial growth of six pathogenic fungi isolated from hill cotton viz., Aspergillus flavus, Aspergillus niger (Type-I), Curvularia lunata, Fusarium moniliforme var. subglutinans, Fusarium sporotrichioides and Rhizoctonia solani. Leaves of the selected plants were collected from the Botanical Garden of Curzon Hall Campus and Kabi Sufia Kamal Hall, University of Dhaka. Leaves of each plant were thoroughly washed in tap water, air dried and then used for fresh extract preparation. Leaf extracts were prepared by crushing known weight of fresh 
leaves with distilled water in ratio of $1: 1(\mathrm{w} / \mathrm{v})$. In this method, the requisite amount of the filtrate of each plant extract was mixed with PDA medium to get 5, 10, 15 and $20 \%$ concentrations.

The medium thus prepared was poured into sterilized Petri plates and allowed to solidify. Each Petri plate was inoculated centrally with a $5 \mathrm{~mm}$ agar disc cut from the margin of actively growing culture of the test pathogens. In the control set, a Petri plate containing PDA medium with the requisite amount of distilled water instead of a plant extract was also inoculated with agar disc of the test pathogen in the same manner as described above. Three replications were maintained for both the experimental and control sets. The inoculated Petri plates were incubated at $25 \pm 2{ }^{\circ} \mathrm{C}$. The radial growth of the colonies of the test pathogen was measured after 5 days of incubation.

The fungitoxicity of the fungicides and plant parts extracts in terms of percentage inhibition of mycelial growth were calculated by using the following formula:

$$
\mathrm{I}=\frac{\mathrm{C}-\mathrm{T}}{\mathrm{C}} \times 100
$$

where, $\mathrm{I}=$ Per cent growth inhibition, $\mathrm{C}=$ Growth in control, $\mathrm{T}=$ Growth in treatment

The data were collected as inhibition percentage of the radial growth of the pathogen in mm in each replication and evaluated by analysis of variance (ANOVA) by using STAR statistical program and means were compared using DMRT.

\section{Results and Discussion}

Twelve species of fungi were isolated from the seeds of three cotton varieties (HC-1, HC-2 and HC-3) following "Tissue planting" and "Blotter" methods during the period of April 2017. The isolated fungi were Aspergillus flavus Link, A. fumigatus Fresenius, A. niger van Tieghem (Type-I), A. niger (Type-II), Chaetomium globosum Kunze ex Fr., Curvularia lunata (Wakker) Boedijn, Fusarium moniliforme var. subglutinans Wr. \& Reink, Fusarium sporotrichioides Sherb., Mem., Penicillium Link, Pestalotiopsis guepinii (Desm.) Stay., Rhizoctonia solani J.G. Kuhn, Rhizopus stolonifer (Ehrenb.: Fr.) Vuill. and Trichoderma viride Pers (Nahar et al. 2019).

Out of 12 isolated fungi six, namely Aspergillus flavus, A. niger (Type-1), Curvularia lunata, Fusarium moniliforme var. subglutinans, Fusarium sporotrichioides and Rhizoctonia solani showed pathogenic potentiality following 'seed inoculation technique'. These pathogenic fungi had remarkable effect on seed germination, root shoot length and mortality of seedlings (Shamsi and Nahar 2019).

Amongst five selected fungicides the complete inhibition of the radial growth of Aspergillus flavus was observed with Autostin 50 WDG and Nativo 75 WP at all the treated concentrations. Capvit 50 WP showed complete growth inhibition of the fungus at 300, 400 and $500 \mathrm{ppm}$ concentrations. The toxicity of these fungicides against Aspergillus flavus at $100 \mathrm{ppm}$ concentration in descending order was Nativo 75 WP / Autostin 50 WDG > Acrobat MZ > Thiovit $80 \mathrm{WG}>$ Capvit $50 \mathrm{WP}$ (Table 1). Out of five fungicides, the complete inhibition of the radial growth of Aspergillus niger (Type-I) was observed in Autostin 50 WDG and Nativo 75 WP at all the treated concentrations. At 100 ppm Capvit 50 WP showed the lowest inhibition (48.7\%). Thiovit 80 WG showed no inhibitory activity at all (Table 1). The toxicity of these fungicides against Aspergillus niger (Type-I) at $100 \mathrm{ppm}$ concentration in descending order was Nativo 75 WP/Autostin $50 \mathrm{WDG}>$ Acrobat MZ > Capvit $50 \mathrm{WP}>$ Thiovit $80 \mathrm{WG}$ (Table 1).

The radial growth of Curvularia lunata was completely inhibited by Nativo $75 \mathrm{WP}$ at all the treated concentrations. Capvit $50 \mathrm{WP}$ showed complete growth inhibition at 400 and $500 \mathrm{ppm}$ 
Table 1. Per cent inhibition of radial growth of pathogenic fungi at different concentrations (ppm) of fungicides.

\begin{tabular}{|c|c|c|c|c|c|c|}
\hline \multirow[t]{2}{*}{ Name of fungi } & \multirow[t]{2}{*}{ Fungicides } & \multicolumn{5}{|c|}{$\begin{array}{l}\% \text { inhibition of radial growth of fungi at different } \\
\text { concentrations (ppm) }\end{array}$} \\
\hline & & 100 & 200 & 300 & 400 & 500 \\
\hline \multirow[t]{6}{*}{ Aspergillus flavus } & Acrobat MZ & $65.19^{b}$ & $69.44^{\mathrm{c}}$ & $72.78^{\mathrm{b}}$ & $75.56^{\mathrm{b}}$ & $79.67^{b}$ \\
\hline & Autostin $50 \mathrm{WDG}$ & $100^{\mathrm{a}}$ & $100^{\mathrm{a}}$ & $100^{\mathrm{a}}$ & $100^{\mathrm{a}}$ & $100^{\mathrm{a}}$ \\
\hline & Capvit $50 \mathrm{WP}$ & $34.62^{\mathrm{d}}$ & $72.12^{\mathrm{b}}$ & $100^{\mathrm{a}}$ & $100^{\mathrm{a}}$ & $100^{\mathrm{a}}$ \\
\hline & Nativo $75 \mathrm{WP}$ & $100^{\mathrm{a}}$ & $100^{\mathrm{a}}$ & $100^{\mathrm{a}}$ & $100^{\mathrm{a}}$ & $100^{\mathrm{a}}$ \\
\hline & Thiovit $80 \mathrm{WG}$ & $51.67^{\mathrm{c}}$ & $59.44^{\mathrm{d}}$ & $61.67^{\mathrm{c}}$ & $64.26^{\mathrm{c}}$ & $68.11^{\mathrm{c}}$ \\
\hline & $\mathrm{CV} \%$ & 1.35 & 1.53 & 0.64 & 0.82 & 1.20 \\
\hline \multirow{6}{*}{$\begin{array}{l}\text { Aspergillus niger } \\
\text { (Type-I) }\end{array}$} & Acrobat MZ & $61.11^{\mathrm{b}}$ & $65.0^{\mathrm{b}}$ & $68.52^{\mathrm{b}}$ & $71.48^{\mathrm{b}}$ & $75.56^{\mathrm{b}}$ \\
\hline & Autostin $50 \mathrm{WDG}$ & $100^{\mathrm{a}}$ & $100^{\mathrm{a}}$ & $100^{\mathrm{a}}$ & $100^{\mathrm{a}}$ & $100^{\mathrm{a}}$ \\
\hline & Capvit 50 WP & $48.7^{\mathrm{c}}$ & $59.44^{\mathrm{c}}$ & $65.0^{c}$ & $68.89^{c}$ & $72.44^{c}$ \\
\hline & Nativo $75 \mathrm{WP}$ & $100^{\mathrm{a}}$ & $100^{\mathrm{a}}$ & $100^{\mathrm{a}}$ & $100^{\mathrm{a}}$ & $100^{\mathrm{a}}$ \\
\hline & Thiovit $80 \mathrm{WG}$ & $0^{\mathrm{d}}$ & $0^{\mathrm{d}}$ & $0^{\mathrm{d}}$ & $0^{\mathrm{d}}$ & $0^{\mathrm{d}}$ \\
\hline & $\mathrm{CV} \%$ & 0.73 & 1.58 & 0.57 & 0.76 & 0.83 \\
\hline \multirow[t]{6}{*}{ Curvularia lunata } & Acrobat MZ & $37.26^{\mathrm{c}}$ & $48.67^{\mathrm{d}}$ & $56.27^{\mathrm{d}}$ & $67.68^{\mathrm{b}}$ & $100^{\mathrm{a}}$ \\
\hline & Autostin $50 \mathrm{WDG}$ & $43.62^{\mathrm{bc}}$ & $53.83^{\mathrm{c}}$ & $59.15^{\mathrm{c}}$ & $64.89^{\mathrm{b}}$ & $70.64^{b}$ \\
\hline & Capvit 50 WP & $48.53^{\mathrm{b}}$ & $66.74^{\mathrm{b}}$ & $80.0^{\mathrm{b}}$ & $100^{\mathrm{a}}$ & $100^{\mathrm{a}}$ \\
\hline & Nativo $75 \mathrm{WP}$ & $100^{\mathrm{a}}$ & $100^{\mathrm{a}}$ & $100^{\mathrm{a}}$ & $100^{\mathrm{a}}$ & $100^{\mathrm{a}}$ \\
\hline & Thiovit $80 \mathrm{WG}$ & $18.6^{\mathrm{d}}$ & $28.29^{\mathrm{e}}$ & $39.15^{\mathrm{e}}$ & $46.89^{c}$ & $56.2^{\mathrm{c}}$ \\
\hline & $\mathrm{CV} \%$ & 6.49 & 3.57 & 1.81 & 2.05 & 3.25 \\
\hline \multirow{6}{*}{$\begin{array}{l}\text { Fusarium } \\
\text { moniliforme var. } \\
\text { subglutinans }\end{array}$} & Acrobat MZ & $37.72^{\mathrm{b}}$ & $46.78^{\mathrm{b}}$ & $58.19^{\mathrm{b}}$ & $64.91^{\mathrm{b}}$ & $70.18^{\mathrm{b}}$ \\
\hline & Autostin $50 \mathrm{WDG}$ & $100^{\mathrm{a}}$ & $100^{\mathrm{a}}$ & $100^{\mathrm{a}}$ & $100^{\mathrm{a}}$ & $100^{\mathrm{a}}$ \\
\hline & Capvit 50 WP & $16.25^{\mathrm{d}}$ & $25.09^{\mathrm{d}}$ & $36.39^{\mathrm{d}}$ & $46.99^{c}$ & $61.84^{\mathrm{c}}$ \\
\hline & Nativo 75 WP & $100^{\mathrm{a}}$ & $100^{\mathrm{a}}$ & $100^{\mathrm{a}}$ & $100^{\mathrm{a}}$ & $100^{\mathrm{a}}$ \\
\hline & Thiovit $80 \mathrm{WG}$ & $27.08^{c}$ & $35.76^{\mathrm{c}}$ & $45.14^{\mathrm{c}}$ & $48.96^{\mathrm{c}}$ & $56.59^{d}$ \\
\hline & $\mathrm{CV} \%$ & 5.16 & 3.66 & 3.11 & 3.43 & 2.51 \\
\hline \multirow{6}{*}{$\begin{array}{l}\text { Fusarium } \\
\text { sporotrichioides }\end{array}$} & Acrobat MZ & $33.96^{\mathrm{b}}$ & $45.28^{\mathrm{b}}$ & $55.47^{\mathrm{b}}$ & $59.62^{\mathrm{b}}$ & $73.58^{\mathrm{b}}$ \\
\hline & Autostin $50 \mathrm{WDG}$ & $100^{\mathrm{a}}$ & $100^{\mathrm{a}}$ & $100^{\mathrm{a}}$ & $100^{\mathrm{a}}$ & $100^{\mathrm{a}}$ \\
\hline & Capvit 50 WP & $25.19^{\mathrm{a}}$ & $30.12^{\mathrm{d}}$ & $44.94^{\mathrm{c}}$ & $61.48^{\mathrm{b}}$ & $100^{\mathrm{a}}$ \\
\hline & Nativo 75 WP & $100^{\mathrm{a}}$ & $100^{\mathrm{a}}$ & $100^{\mathrm{a}}$ & $100^{\mathrm{a}}$ & $100^{\mathrm{a}}$ \\
\hline & Thiovit $80 \mathrm{WG}$ & $27.88^{\mathrm{bc}}$ & $35.89^{\mathrm{c}}$ & $43.27^{\mathrm{c}}$ & $50.96^{\mathrm{c}}$ & $56.09^{c}$ \\
\hline & $\mathrm{CV} \%$ & 7.01 & 3.68 & 3.39 & 3.10 & 1.13 \\
\hline \multirow[t]{6}{*}{ Rhizoctonia solani } & Acrobat MZ & $16.22^{\mathrm{b}}$ & $31.89^{\mathrm{b}}$ & $35.78^{\mathrm{b}}$ & $38.89^{c}$ & $46.89^{c}$ \\
\hline & Autostin $50 \mathrm{WDG}$ & $100^{\mathrm{a}}$ & $100^{\mathrm{a}}$ & $100^{\mathrm{a}}$ & $100^{\mathrm{a}}$ & $100^{\mathrm{a}}$ \\
\hline & Capvit $50 \mathrm{WP}$ & $0^{\mathrm{c}}$ & $20.33^{\mathrm{d}}$ & $31.11^{\mathrm{c}}$ & $68.67^{\mathrm{b}}$ & $83.56^{\mathrm{b}}$ \\
\hline & Nativo $75 \mathrm{WP}$ & $100^{\mathrm{a}}$ & $100^{\mathrm{a}}$ & $100^{\mathrm{a}}$ & $100^{\mathrm{a}}$ & $100^{\mathrm{a}}$ \\
\hline & Thiovit 80 WG & $16.33^{\mathrm{b}}$ & $25.78^{\mathrm{c}}$ & $31.11^{\mathrm{c}}$ & $33.33^{\mathrm{d}}$ & $36.67^{\mathrm{d}}$ \\
\hline & $\mathrm{CV} \%$ & 0.71 & 4.96 & 2.68 & 1.28 & 0.73 \\
\hline
\end{tabular}

Means followed by the same letter within a column did not differ significantly at $5 \%$ level by DMRT. 
concentrations. Acrobat MZ showed complete inhibition at $500 \mathrm{ppm}$ concentration. The lowest activity (18.6\%) was shown by Thiovit $80 \mathrm{WG}$ at $100 \mathrm{ppm}$ concentration (Table 1). The toxicity of these fungicides against Curvularia lunata at $100 \mathrm{ppm}$ concentration in descending order was Nativo 75 WP > Capvit 50 WP > Autostin 50 WDG > Acrobat MZ > Thiovit 80 WG (Table 1). In Fusarium moniliforme var. subglutinans the complete inhibition was observed with Autostin 50 WDG and Nativo $75 \mathrm{WP}$ at all the treated concentrations. At $500 \mathrm{ppm}$ concentration Acrobat MZ, Capvit 50 WP and Thiovit $80 \mathrm{WG}$ showed 70.18, 61.84 and 56.59\% radial growth inhibitions, respectively. The toxicity of these fungicides against Fusarium moniliforme var. subglutinans at $100 \mathrm{ppm}$ concentration in descending order was Nativo $75 \mathrm{WP} /$ Autostin $50 \mathrm{WDG}>$ Acrobat MZ $>$ Thiovit $80 \mathrm{WG}>$ Capvit $50 \mathrm{WP}$ (Table 1). The radial growth of Fusarium sporotrichioides was completely inhibited by Autostin 50 WDG and Nativo $75 \mathrm{WP}$ at all the treated concentrations. Capvit $50 \mathrm{WP}$ showed complete growth inhibition at $500 \mathrm{ppm}$ concentrations. The toxicity of these fungicides against Fusarium sporotrichioides at $100 \mathrm{ppm}$ concentration in descending order was Nativo $75 \mathrm{WP} /$ Autostin $50 \mathrm{WDG}>$ Acrobat MZ > Thiovit $80 \mathrm{WG}>$ Capvit $50 \mathrm{WP}$ (Table 1).

In Rhizoctonia solani the complete inhibition was observed with Autostin 50 WDG and Nativo $75 \mathrm{WP}$ at all the treated concentrations. At $500 \mathrm{ppm}$ concentration Acrobat MZ, Capvit 50 WP and Thiovit 80 WG showed $46.89,83.56$ and $36.67 \%$ radial growth inhibitions, respectively. The toxicity of these fungicides against Rhizoctonia solani at $100 \mathrm{ppm}$ concentration in descending order was Nativo $75 \mathrm{WP} /$ Autostin $50 \mathrm{WDG}>$ Thiovit $80 \mathrm{WG}>$ Acrobat MZ > Capvit $50 \mathrm{WP}$ (Table 1). It is also apparent from the results that the per cent growth inhibition of the test pathogens gradually increased with the increase in concentration of the fungicides. Khatun and Shamsi (2016) found that Bavistin $50 \mathrm{WP}$ and Greengel $72 \mathrm{WP}$ showed complete radial growth inhibition of Aspergillus flavus and Curvularia lunata at 400 and $500 \mathrm{ppm}$ concentrations. The present investigation was similar with aforesaid experiment. Rathod and Pawar (2013) reported that the combination of Mancozeb and Cupravit $50 \mathrm{WP}$ both at $0.4 \%$ significantly reduced the mycelial growth of Fusarium spp, Alternaria spp, Sclerotium spp, Aspergillus flavus and A. niger after seven days of observation. Previously it was reported that bavistin, mancozeb, carbindazim, hexacanazole, cupravit and benlate inhibited the radial growth of Fusarium spp. (Fravel et al. 2005, Iqbal et al. 2010, Chowdhury et al. 2015, Mamun et al. 2016).

Out of the five plant extracts, Psidium guajava showed highest $(81.29 \%)$ radial growth inhibition of Aspergillus flavus at 20\% concentration which was followed by Azadirachta indica (78.11\%), Agle mermelos (68.33\%), and Adhatoda vasica (64.91\%). Dutura metel showed no inhibition. The order of effectiveness of plant extracts against Aspergillus flavus at $20 \%$ concentration was Psidium guajava > Azadirachta indica $>$ Aegle mermelos $>$ Adhatoda vasica $>$ Datura metel (Table 2). Azadirachta indica showed maximum (65.56\%) radial growth inhibition of Aspergillus niger (Type-I) at $20 \%$ concentration which was followed by Dutura metel (63.67\%), Agle mermelos (60.78\%), and A. vasica (58.70\%). There was no growth inhibition of A. niger (Type-I) by Psidium guajava. The order of effectiveness of plant extracts against Aspergillus niger (Type-I) at 20\% concentration was Azadirachta indica > Datura metel $>$ Aegle mermelos $>$ Adhatoda vasica > Psidium guajava (Table 2).

The highest inhibition of the radial growth of Curvularia lunata was observed with Psidium guajava (72.26\%) at $20 \%$ concentration which was followed by A. indica (61.90\%), Dutura metel (61.83\%), A. vasica (47.14\%) and Aegle mermelos (44.02\%). The order of effectiveness of plant extracts against Curvularia lunata at 20\% concentration was Psidium guajava > Azadirachta indica $>$ Datura metel $>$ Adhatoda vasica $>$ Aegle mermelos (Table 2). 
Table 2. Per cent inhibition of radial growth of pathogenic fungi at different concentrations of plant extracts.

\begin{tabular}{|c|c|c|c|c|c|}
\hline \multirow[t]{2}{*}{$\begin{array}{l}\text { Name of } \\
\text { fungi }\end{array}$} & \multirow[t]{2}{*}{$\begin{array}{l}\text { Name of } \\
\text { plants }\end{array}$} & \multicolumn{4}{|c|}{$\begin{array}{l}\% \text { inhibition of radial growth of fungi at } \\
\text { different concentrations }\end{array}$} \\
\hline & & 5 & 10 & 15 & 20 \\
\hline \multirow[t]{6}{*}{ Aspergillus flavus } & Adhatoda vasica Nees. & $52.88^{\mathrm{b}}$ & $55.14^{\mathrm{d}}$ & $60.15^{\mathrm{c}}$ & $64.91^{\mathrm{d}}$ \\
\hline & Aegle mermelos L. & $45.56^{\mathrm{c}}$ & $58.33^{\mathrm{c}}$ & $62.78^{\mathrm{c}}$ & $68.33^{\mathrm{c}}$ \\
\hline & Azadirachta indica A. Juss. & $58.0^{\mathrm{a}}$ & $63.11^{\mathrm{b}}$ & $65.78^{\mathrm{b}}$ & $78.11^{\mathrm{b}}$ \\
\hline & Datura metel L. & $0^{\mathrm{d}}$ & $0^{\mathrm{e}}$ & $0^{\mathrm{d}}$ & $0^{\mathrm{e}}$ \\
\hline & Psidium guajava $\mathrm{L}$. & $62.22^{\mathrm{a}}$ & $70.22^{\mathrm{a}}$ & $76.11^{\mathrm{a}}$ & $81.29^{\mathrm{a}}$ \\
\hline & $\mathrm{CV}(\%)$ & 6.40 & 2.56 & 2.93 & 2.21 \\
\hline \multirow{6}{*}{$\begin{array}{l}\text { Aspergillus niger } \\
\text { (Type-I) }\end{array}$} & Adhatoda vasica Nees. & $0^{\mathrm{b}}$ & $36.11^{\mathrm{b}}$ & $52.59^{\mathrm{b}}$ & $58.70^{\mathrm{b}}$ \\
\hline & Aegle mermelos L. & $22.0^{\mathrm{a}}$ & $47.0^{\mathrm{a}}$ & $53.56^{\mathrm{b}}$ & $60.78^{\mathrm{ab}}$ \\
\hline & Azadirachta indica A. Juss. & $0^{\mathrm{b}}$ & $50.0^{\mathrm{a}}$ & $58.0^{\mathrm{a}}$ & $65.56^{\mathrm{a}}$ \\
\hline & Datura metel L. & $0^{\mathrm{b}}$ & $32.56^{\mathrm{b}}$ & $51.89^{\mathrm{b}}$ & $63.67^{\mathrm{ab}}$ \\
\hline & Psidium guajava $\mathrm{L}$. & $0^{\mathrm{b}}$ & $0^{\mathrm{c}}$ & $0^{\mathrm{c}}$ & $0^{\mathrm{c}}$ \\
\hline & $\mathrm{CV}(\%)$ & 32.10 & 12 & 2.46 & 6.47 \\
\hline \multirow[t]{6}{*}{ Curvularia lunata } & Adhatoda vasica Nees. & $21.15^{\mathrm{c}}$ & $29.07^{\mathrm{cd}}$ & $37.44^{\mathrm{c}}$ & $47.14^{\mathrm{c}}$ \\
\hline & Aegle mermelos L. & $20.94^{\mathrm{c}}$ & $26.34^{\mathrm{d}}$ & $30.34^{\mathrm{d}}$ & $44.02^{\mathrm{c}}$ \\
\hline & Azadirachta indica A. Juss & $30.16^{\mathrm{b}}$ & $34.29^{\mathrm{bc}}$ & $49.84^{\mathrm{b}}$ & $61.90^{\mathrm{b}}$ \\
\hline & Datura metel L. & $32.26^{\mathrm{b}}$ & $38.17^{\mathrm{b}}$ & $43.55^{\mathrm{bc}}$ & $61.83^{\mathrm{b}}$ \\
\hline & Psidium guajava $\mathrm{L}$. & $47.26^{\mathrm{a}}$ & $54.45^{\mathrm{a}}$ & $59.93^{\mathrm{a}}$ & $72.26^{\mathrm{a}}$ \\
\hline & $\mathrm{CV}(\%)$ & 9.55 & 8.93 & 7.86 & 5.29 \\
\hline \multirow{6}{*}{$\begin{array}{l}\text { Fusarium } \\
\text { moniliforme var. } \\
\text { subglutinans }\end{array}$} & Adhatoda vasica Nees. & $28.95^{\mathrm{c}}$ & $34.21^{\mathrm{c}}$ & $46.99^{c}$ & $54.14^{\mathrm{c}}$ \\
\hline & Aegle mermelos L. & $21.9^{\mathrm{d}}$ & $29.75^{\mathrm{d}}$ & $34.71^{\mathrm{d}}$ & $47.52^{\mathrm{d}}$ \\
\hline & Azadirachta indica A. Juss & $49.61^{\mathrm{a}}$ & $57.42^{\mathrm{a}}$ & $64.84^{\mathrm{a}}$ & $75.0^{\mathrm{a}}$ \\
\hline & Datura metel L. & $21.9^{\mathrm{d}}$ & $29.75^{\mathrm{d}}$ & $34.71^{\mathrm{d}}$ & $47.52^{\mathrm{d}}$ \\
\hline & Psidium guajava $\mathrm{L}$. & $41.24^{\mathrm{b}}$ & $49.48^{\mathrm{b}}$ & $54.64^{\mathrm{b}}$ & $64.95^{\mathrm{b}}$ \\
\hline & $\mathrm{CV}(\%)$ & 9.31 & 5.74 & 6.20 & 3.59 \\
\hline \multirow{6}{*}{$\begin{array}{l}\text { Fusarium } \\
\text { sporotrichioides }\end{array}$} & Adhatoda vasica Nees. & $30.85^{\mathrm{a}}$ & $40.43^{\mathrm{b}}$ & $44.68^{\mathrm{b}}$ & $62.77^{\mathrm{a}}$ \\
\hline & Aegle mermelos L. & $9.27^{\mathrm{b}}$ & $19.51^{\mathrm{d}}$ & $24.88^{\mathrm{c}}$ & $38.54^{\mathrm{c}}$ \\
\hline & Azadirachta indica A. Juss. & $31.45^{\mathrm{a}}$ & $36.02^{\mathrm{bc}}$ & $39.52^{\mathrm{b}}$ & $44.89^{\mathrm{b}}$ \\
\hline & Datura metel L. & $27.05^{\mathrm{a}}$ & $35.59^{c}$ & $40.93^{\mathrm{b}}$ & $64.77^{\mathrm{a}}$ \\
\hline & Psidium guajava $\mathrm{L}$. & $25.93^{\mathrm{a}}$ & $46.8^{\mathrm{a}}$ & $56.57^{\mathrm{a}}$ & $62.63^{\mathrm{a}}$ \\
\hline & $\mathrm{CV}(\%)$ & 17.62 & 7.54 & 7.98 & 4.07 \\
\hline \multirow[t]{6}{*}{ Rhizoctonia solani } & Adhatoda vasica Nees. & $0^{\mathrm{b}}$ & $0^{\mathrm{b}}$ & $0^{\mathrm{b}}$ & $0^{\mathrm{b}}$ \\
\hline & Aegle mermelos L. & $0^{\mathrm{b}}$ & $0^{\mathrm{b}}$ & $0^{\mathrm{b}}$ & $0^{\mathrm{b}}$ \\
\hline & Azadirachta indica A. Juss. & $0^{\mathrm{b}}$ & $0^{\mathrm{b}}$ & $0^{\mathrm{b}}$ & $0^{\mathrm{b}}$ \\
\hline & Datura metel L. & $0^{\text {constant }}$ & $20.56^{\mathrm{a}}$ & $37.56^{\mathrm{a}}$ & $42.44^{\mathrm{a}}$ \\
\hline & Psidium guajava $\mathrm{L}$. & $0^{\mathrm{b}}$ & $0^{\mathrm{b}}$ & $0^{\mathrm{b}}$ & $0^{\mathrm{b}}$ \\
\hline & $\mathrm{CV}(\%)$ & Constant & 16 & 10.09 & 3.38 \\
\hline
\end{tabular}

Means followed by the same letter within a column did not differ significantly at 5\% level by DMRT.

The highest inhibition of the radial growth of Fusarium moniliforme var. subglutinans was observed with Azadirachta indica $(75.0 \%)$ at $20 \%$ concentration which was followed by Psidium 
guajava (64.95\%), A. vasica (54.14\%), Dutura metel (47.52\%), and Aegle mermelos (47.52\%). The order of effectiveness of plant extracts against Fusarium moniliforme var. subglutinans at $20 \%$ concentration was Azadirachta indica > Psidium guajava > Adhatoda vasica > Aegle mermelos > Datura metel (Table 2). The highest inhibition of the radial growth of Fusarium sporotrichioides was observed with Dutura metel (64.77\%) at 20\% concentration which was followed by Adhatoda visica (62.77\%), Psidium guajava (62.63\%), A. indica (44.89\%), and Aegle mermelos (38.54\%). The order of effectiveness of plant extracts against Fusarium sporotrichioides at $20 \%$ concentration was Datura metel > Adhatoda vasica $>$ Psidium guajava > Azadirachta indica > Aegle mermelos (Table 2). Exclusively Dutura metel showed inhibition of the radial growth of Rhizoctonia solani at 10, 15 and $20 \%$ and the inhibition percentage was 20.56, 37.56 and 42.44 (Table 2).

Mondall et al. (2009) reported that the crude aqueous and alcoholic leaf extracts of Azadirachta indica was more effective in inhibitions of growth of the fungus Aspergillus in comparison to inhibitory effects on Rhizopus growth in the artificial culture medium. Shanaz et al. (2010) reported the inhibitory effect of Datura alba against Macrophomina phaseolina and Rhizoctonia solani. Khatun and Shamsi (2016) reported that the complete inhibition of radial growth of Curvularia lunata was observed with plant extract of A. indica and D. metel at $20 \%$ concentration. William (2008) reported that Lowsonia inermis inhibited conidial germination of A. flavus and A. fumigatus while A. niger was mostly inhibited by A. indica. Mamun et al. (2016) reported that Azadirachta indica and D. metel showed promising activity against Fusarium spp. The present result also showed similar activity.

The present investigation suggests that out of five fungicides Nativo 75 WP and Autostin 50 WDG are identified as the best inhibiting fungicides for six pathogenic fungi of cotton seeds and out of five plant extracts, depending on the pathogenic fungi Azadirachta indica, Datura metel and Psidium guajava showed promising effect.

\section{Acknowledgements}

The first author (MNN) gratefully acknowledges the financial support by the Ministry of Science and Technology, Government of the People's Republic of Bangladesh through NST fellowship.

\section{References}

Bansal KR and Rajesh KG 2000. Evaluation of plant extracts against Fusarium oxysporum, wilt pathogen of fenugreek. Indian. J. Phytopath. 53(1): 107-108.

Barnett HL and Hunter BB 2000. Illustrated Genera of Imperfect Fungi. 4th edn., Burgess Pub. Co. Minneapolis. pp. 185.

Benoit MA and Mathur SB 1970. Identification of species Curvularia on rice seed. Proc. Inst. Seed Test. Ass. 35(1): 1-23.

Booth C 1971. The genus Fusarium. Commonwealth Mycological Institute, Kew, Surrey, England. pp. 237.

Chowdury P, Bashar MA and Shamsi S 2015. In vitro evaluation of fungicides and plant extracts against pathogenic fungi of two rice varieties in Bangladesh. Bangladesh J. Bot. 24(2): 251-259.

Fravel DR, Deahl KC and Stommel JR 2005. Capability of biocontrol fungus Fusarium oxysporum strain CS20 with selected chemicals. Biol. Cont. 34: 165-169.

Hillocks RJ, Chinodya R and Gunner R 1988. Evaluation of seed dressing and in-furrow treatments with fungicides for control of seedling disease in cotton caused by Rhizoctonia solani. Crop Prot. 7: 309-313. 
Iqbal Z, Pervez MA, Ahmad S, Iftikhar Y, Yasin M, Nawaz A, Ghazanfar MU, Dasti AA and Saleem A 2010. Determination of minimum inhibitory concentrations of fungicides against fungus Fusarium mangiferae. Pak. J. Bot. 42(5): 3525-3532.

Johnson LF and Chambers AY 1973. Isolation and identity of three species of Pythium that cause cotton seedling blight. Plant Dis. Rep. 57: 848-852.

Khatun A and Shamsi S 2016. In vitro evaluation of fungicides and plant extracts against the fungi associated with seeds of nine chickpea varieties. Dhaka Univ. J. Biol. Sci. 25(1): 83-90.

Mamun MA, Shamsi S and Bashar MA 2016. In vitro evaluation of fungicides and plant extracts against pathogenic fungi of jute seeds. Biores. Comm. 2(1): 189-192.

McLean KS and Gazaway WS 2000. Evaluation of selected in-furrow fungicides and fungicide biological combinations for control of seedling disease in cotton. In: 1999 Cotton Research Report, Alabama Agricultural Experiment Station, Research Report Series No. 18, Auburn, AL. p. 40.

Mondall NK, Mojumdar A, Chatterje SK, Banerjee A, Datta JK and Gupta S 2009. Antifungal activities and chemical characterization of Neem leaf extracts on the growth of some selected fungal species in vitro culture medium. J. Appl. Sci. Environ. 13(1): 49-53.

Nahar MN, Hosen S and Shamsi S 2019. Prevalence of fungi associated with seeds of three cotton varieties (Gossypium arboreum L.) in storage. Bioresearch Communications 5(1): 642-648.

Shamsi S and Nahar MN 2019. Pathogenic potentiality of fungi isolated from three varieties of hill cotton seeds (Gossypium arboreum L.). Dhaka Univ. J. Biol. Sci. 28(2): 1487-193.

Pandey BP 1980. Economic Botany: Fiber and Fiber Yielding Plants. pp. 138-147.

Rathod LR and Pawar NB 2013. In vitro seed treatment of Fungicides for the control of seed borne fungi of soyabean variety Durga. Global Research Analysis 2(10):15-16.

Sayago JE, Ordoñez RM, Kovacevich LN, Torres S and Isla MI 2012. Antifungal activity of extracts of extremophile plants from the Argentine Puna to control citrus postharvest pathogens and green mold. Postharvest Biology and Technology 67: 19-24.

Shahnaz D, Sadia K and Marium T 2010. Comparative effect of plant extract of Datura alba Nees and Cynodon dactylon (L.) Pers., alone or in combination with microbial antagonists for the control of root rot disease of cowpea and okra. Pakistan Journal of Botany 42(2): 1273-1279.

Shamsi S, Nahar N, Chaowdhury P and Momtaz S 2010. Fungal diseases of three aromatic rice (Oryza sativa L.). Journal of Bangladesh Academy of Sciences 34(2): 63-70.

So ML 1990. Antifungal activities of mangrove plants. Proceedings of the 3rd International Conference on Plant Protection in the Tropics. Malaysian Plant Protection Society Malaysia 2: 95-98.

Thom C and Raper KB 1945. A manual of the Aspergilli. Williams and Wilkins, Baltimore, M.D. USA. pp. 373.

Uddin FM and Mortuza MG 2015. Cotton production in Bangladesh: Current scenario and prospect. Bangladesh Rural Advancement Committee, Bangladesh. pp. 10.

Varma J and Dubey NK 1999. Prospectives of botanical and microbial products as pesticides of tomorrow. Curr. Sci. 25: 172-179.

Vollmann J and Laimer M 2013. Novel and traditional oil crops and their biorefinery potential. Bioprocessing Technologies in Biorefinery for Sustainable Production of Fuels, Chemicals and Polymers 12: 47-60.

Wendel JF, Brubaker C, Alvarez I, Cronn R and Stewart JM 2009. Evolution and natural history of the cotton genus. In: Andrew H. Paterson (Ed.). Genetics and Genomics of Cotton. Plant Genetics and Genomics: Crops and Models 3: 3-22.

William Q 2008. Least toxic controls of plant diseases. Brooklyn Botanic garden. Natural Disease Control 11: 225 . 